\title{
Mecanismos da Interação Antígeno-anticorpo em uma Resposta Primária Célula T-mediada ${ }^{1}$
}

S. BARROZO2 ${ }^{2}$ Departamento de Físico-Química, Instituto de Química, UNESP, Rua Prof. Francisco Degni s/n, 14800-900 Araraquara, SP, Brasil

H.M. YANG ${ }^{3}$, Departamento de Matemática Aplicada, Instituto de Matemática, Estatística e Computação Científica, UNICAMP, Cx.P. 6065, 13081-970 Campinas, SP, Brasil.

Resumo. Os mecanismos de ação das células $\mathrm{T}$, mediante a presença de um antígeno no organismo, são essenciais para a produção de anticorpos no combate a patógenos circulantes na corrente sanguínea. Este trabalho estuda um modelo matemático que explora os mecanismos de produção de anticorpos pelas células $\mathrm{B}$, mediante a presença de um antígeno no organismo e mediada pela ação das células $\mathrm{T}$, bem como a ação dos anticorpos. O modelo é composto por um sistema de equações diferenciais ordinárias não-lineares de primeira ordem, o qual descreve a interação das células $\mathrm{B}$ e $\mathrm{T}$ do sistema imunológico entre si e com o antígeno. O estudo das soluções em equilíbrio estacionário mostra a existência de uma região de parâmetros na qual três soluções de equilíbrio são biologicamente viáveis, sendo duas estáveis, as quais representam, respectivamente, a ausência de antígeno (solução trivial) e a sua existência em concentração maior, e uma intermediária instável, com concentração menor de antígenos, denominada "break point". Fora desta região, apenas a solução trivial é biologicamente viável. Isso evidencia a resposta do sistema imunológico depender tanto do seu estado quanto da concentração de antígenos inoculados, concordando com o observado na prática.

\section{Introdução}

O sistema imunológico, em condições ideais, está preparado para responder a todas as possibilidades de ataques de organismos estranhos ao indivíduo, sejam por micro ou macro-parasitas, células cancerígenas, ou qualquer substância que não seja reconhecida como integrante da composição química do hospedeiro. Todavia, diante da grande diversidade de elementos estranhos, faz-se necessária também uma grande variedade de mecanismos de defesa e de células envolvidas em cada um deles, a fim de se garantir o sucesso do defensor na batalha. E a evolução das espécies mais complexas garantiu esta possibilidade. O sistema imunológico é, portanto,

\footnotetext{
${ }^{1}$ Apoio financeiro CNPq (Edital Universal 01/2002)

${ }^{2}$ sbarrozo@iq.unesp.br, apoio financeiro FUNDUNESP

${ }^{3}$ hyunyang@ime.unicamp.br, apoio financeiro CNPq
} 
um sistema complexo capaz de identificar e responder de modo específico a cada elemento estranho ao organismo. A descrição de sua atuação é bem documentada na literatura e pode ser vista, por exemplo, em [5] [6] [7] [9] [10] [11] [13].

A ação de sistema imunológico sobre micro-organismos circulantes na corrente sangüínea, ou em regiões extra-celulares, pode-se dar através de resposta imunológica inata ou por ação de macrófagos. Porém uma resposta imunológica denominada humoral, na qual a ação de anticorpos é fundamental, é mais eficaz do que as anteriores. Os anticorpos, ao se ligarem aos antígenos, sinalizam aos macrófagos ou ao sistema de complementos que aquele elemento deve ser destruído, assim como pode perfurar a membrana celular do patógeno, facilitando sua eliminação, ou ainda, impedir que as toxinas produzidas pelo patógeno afetem o hospedeiro [6]. Todavia, a produção de anticorpos só se dá mediante um estímulo antigênico. De modo suscinto, pode-se dizer que um antígeno circulante é detectado por células ditas apresentadoras de antígeno, as quais podem ser células dentríticas, macrófagos, monócitos ou células B. Estas células atam-se ao antígeno e migram para algum órgão linfóide secundário, como basso, amígdalas e apêndice, dentre outros, onde ocorre a resposta imunológica. Uma vez nestes órgãos, a célula apresentadora de antígeno ata-se a uma célula $T$ auxiliadora em estado de repouso, a qual é ativada e, após confirmar que o elemento realmente é estranho ao organismo, se prolifera e produz citocinas que serão essenciais na produção de anticorpos. Estas células, ditas $T$ ativadas, ao encontrarem células $B$ que possuam receptores para este antígeno e o tenham encontrado, unem-se a elas, as quais se tornam ativadas e se diferenciam em células denominadas plasma. Estas últimas, através do efeito de citocinas especiais liberadas pelas células $T$ ativadas, se dividem por processo de clonagem e produzem milhares de anticorpos, os quais são liberados na corrente sanguínea e irão se ligar aos patógenos a fim de promover sua eliminação [1].

Um modelo matemático que descreve a interação entre antígeno e sistema imunológico em uma resposta primária foi desenvolvido em [3], o qual leva em conta a ação das células apresentadoras de antígeno, das células $B$ e das células $T$ nas formas auxiliadoras, supressoras e citotóxicas. A simplificação daquele modelo complexo

culminou no modelo estudado aqui, o qual explora os mecanismos da interação antígeno-anticorpo mediada pela ação das células $B$ e $T$.

\section{O Modelo}

O modelo apresentado em [3] considera antígenos circulantes na corrente sanguínea, sejam por recente contato com o hospedeiro ou por multiplicação de patógenos em suas células ou tecidos. Estes antígenos, designados por $A$, representam microorganismos que tenham invadido um hospedeiro e que se replicam a uma taxa intrínseca $k_{A}$, sendo que sua multiplicação está limitada ou pela disponibilidade das células alvo, onde ocorrem as sínteses protéicas para a sua replicação, ou pelos suprimentos para bactérias extra-celulares. Esta disponibilidade é designada por $C$ e representa a capacidade de suporte do meio. Considera-se que parte destes microorganismos são inviabilizados naturalmente (replicação defeituosa ou dificuldade em encontrar células-alvo) a uma taxa de "mortalidade" $\mu_{A}$, e parte deles são 
debelados do organismo, devido à ação do sistema imune, a uma taxa $\alpha$. É sabido que a eliminação de antígenos se dá principalmente através de seu encontro com anticorpos produzidos para este fim, porém, como a concentração de anticorpos é proporcional à quantidade de células plasma que os produziu $\left(B_{p}\right)$, o modelo considera que o encontro de uma célula $B_{p}$ com o antígeno provoca a morte deste.

A resposta imunológica é representada pela atuação das células $B$ e $T$, sendo considerado que o encontro de uma célula $T$ com um antígeno pode resultar na ativação desta célula $T$, o que ocorre a uma taxa $\delta$. O processo de apresentação do antígeno à célula $T$, via células apresentadoras de antígenos, bem como o mecanismo de reconhecimento de um organismo estranho que deve ser eliminado, estão implícitos no modelo através da taxa de ativação das células $T$, mediante o seu encontro com $A$. As células $T$ ativadas $\left(T_{a}\right)$, ao encontrarem as células $B$ que também detectaram o mesmo antígeno, provocam a ativação destas últimas, a uma taxa $\beta$, as quais se diferenciam em células plama. Estas, através do estímulo de citocinas produzidas pelas células $T$ ativadas, dividem-se por um processo de clonagem, a uma taxa $\gamma$, e produzem anticorpos a fim de eliminar o patógeno. Considerando-se a homeostasia, assume-se que as células $B$ são produzidas pela medula óssea a uma taxa constante $k_{B}$, enquanto que as células $T$ são produzidas no timo a uma taxa constante $k_{T}$, e morrem com taxas constantes $\mu_{B}$ e $\mu_{T}$, respectivamente.

Considerando as hipóteses acima, a interação entre sistema imunológico e parasita pode ser descrita através do seguinte sistema de equações diferenciais ordinárias

$$
\left\{\begin{array}{l}
\frac{d A}{d t}=k_{A}\left(1-\frac{A}{C}\right) A^{2}-\mu_{A} A-\alpha A B_{p} \\
\frac{d B}{d t}=k_{B}-\mu_{B} B-\beta T_{a} B \\
\frac{d B_{p}}{d t}=\beta T_{a} B-\mu_{B}^{\prime} B_{p}+\gamma T_{a} B_{p} \\
\frac{d T}{d t}=k_{T}-\mu_{T} T-\delta A T \\
\frac{d T_{a}}{d t}=\delta A T-\mu_{T}^{\prime} T_{a},
\end{array}\right.
$$

onde $\mu_{B}^{\prime}$ e $\mu_{T}^{\prime}$ são as taxas de mortalidade, respectivamente, das células $B$ e $T$ ativadas, as quais englobam mortalidade natural mais apoptose, uma vez que o modelo considera que as células ativadas, após cumprirem seu papel, morrem por apoptose (morte celular programada). A função $k_{A}\left(1-\frac{A}{C}\right) A$ é utilizada para representar a taxa de crescimento per-capita dos parasitas. A ação de anticorpos (produzidos pelas células $B$ plasma) sobre antígenos é descrita pelo termo $\alpha A B_{p}$. As células $B$ ativadas multiplicam-se proporcionalmente à concentração de células $T$ ativadas, devido à ação de suas citocinas, o que é descrito pelo termo $\gamma T_{a} B_{p}$, assim como a ativação das células $T$ é proporcional à concentração de antígenos, descrito pelo termo $\delta A T$. O modelo apresentado em [2] considerou que as células plasma

$B_{p}$ proliferam pela presença de antígenos. As variáveis dinâmicas têm dimensão [concentração $] \times\left[\mathrm{mm}^{3}\right]^{-1}$.

O estudo dos pontos de equilíbrio estacionário do modelo descrito pelo sistema de equações (2.1) é divido em duas partes.

\subsection{Equilíbrio trivial}

O ponto de equilíbrio trivial do sistema de equações (2.1), o qual corresponde ao sistema imunológico em repouso, representando a situação de ausência de antígeno, 
ou por não ter ocorrido infecção ou subseqüente a uma resposta imunológica bem sucedida, é dado por $P_{0}=\left(\bar{A}, \bar{B}, \bar{B}_{p}, \bar{T}, \bar{T}_{a}\right)$, onde $\bar{B}=\frac{k_{B}}{\mu_{B}}, \bar{T}=\frac{k_{T}}{\mu_{T}}$ e $\bar{A}=\bar{B}_{p}=$ $\bar{T}_{a}=0$.

A estabilidade do ponto de equilíbrio trivial $P_{0}$ é dada pelos auto-valores associados à matriz Jacobiana (linearização do sistema dinâmico em torno do valor de equilíbrio), a qual é dada por

$$
J=\left[\begin{array}{ccccc}
-\mu_{A} & 0 & 0 & 0 & 0 \\
0 & -\mu_{B} & 0 & 0 & -\beta B \\
0 & 0 & -\mu_{B}^{\prime} & 0 & \beta B \\
-\delta T & 0 & 0 & -\mu_{T} & 0 \\
\delta T & 0 & 0 & 0 & -\mu_{T}^{\prime}
\end{array}\right]
$$

Os auto-valores associados à matriz Jacobiana no ponto de equilíbrio trivial são $\lambda_{1}=-\mu_{A}, \lambda_{2}=-\mu_{B}, \lambda_{3}=-\mu_{B}^{\prime}, \lambda_{4}=-\mu_{T}$ e $\lambda_{5}=-\mu_{T}^{\prime}$. Como todos os autovalores são negativos [4], segue que a solução de equilíbrio trivial é localmente e assintoticamente estável (L.A.E.). Isso significa que um dos atratores do sistema dinâmico representa a eliminação do parasita, em que o sistema imunológico reassume o estado de repouso e fica em prontidão contra novas e outras infecções.

Assim, o equilíbrio trivial $P_{0}$ é L.A.E., independentemente dos parâmetros do modelo. Isto implica que, sob determinadas condições, ou mesmo na ausência de vínculos, o sistema dinâmico tende à situação em que a infecção é debelada.

\subsection{Equilíbrio não-trivial}

O equilíbrio não-trivial do sistema de equações (2.1), o qual representa a co-existência de todas as variáveis que compõem o modelo, ou seja, a presença do antígeno juntamente com todas as células do sistema imunológico que estão sendo consideradas, é dado por $P=\left(\bar{A}, \bar{B}, \bar{B}_{p}, \bar{T}, \bar{T}_{a}\right)$, onde

$$
\left\{\begin{array}{l}
\bar{B}=\frac{k_{B} \mu_{T}^{\prime}\left(\mu_{T}+\delta \bar{A}\right)}{\mu_{B} \mu_{T}^{\prime}\left(\mu_{T}+\delta \bar{A}\right)+\beta \delta k_{T} \bar{A}} \\
\bar{B}_{p}=\frac{1}{\alpha}\left[k_{A}\left(1-\frac{\bar{A}}{C}\right) \bar{A}-\mu_{A}\right] \\
\bar{T}=\frac{k_{T}}{\mu_{T}+\delta \bar{A}} \\
\bar{T}_{a}=\frac{\delta k_{T} \bar{A}}{\mu_{T}^{\prime}\left(\mu_{T}+\delta \bar{A}\right)}
\end{array}\right.
$$

sendo que o valor de $\bar{A}$ é obtido da solução da equação

$$
c_{4} \bar{A}^{4}+c_{3} \bar{A}^{3}+c_{2} \bar{A}^{2}+c_{1} \bar{A}+c_{0}=0
$$


onde os coeficientes do polinômio do quarto grau são dados por

$$
\left\{\begin{aligned}
c_{4}= & \delta^{2} k_{A}\left(\mu_{B} \mu_{T}^{\prime}+\beta k_{T}\right)\left(\gamma k_{T}-\mu_{B}^{\prime} \mu_{T}^{\prime}\right) \\
c_{3}= & k_{A} \delta\left(\gamma k_{T}-\mu_{B}^{\prime} \mu_{T}^{\prime}\right)\left[\mu_{B} \mu_{T} \mu_{T}^{\prime}-\delta C\left(\mu_{B} \mu_{T}^{\prime}+\beta k_{T}\right)\right] \\
& -\delta k_{A} \mu_{B}^{\prime} \mu_{T} \mu_{T}^{\prime}\left(\mu_{B} \mu_{T}^{\prime}+\beta k_{T}\right) \\
c_{2}= & \delta C\left(\gamma k_{T}-\mu_{B}^{\prime} \mu_{T}^{\prime}\right)\left[\mu_{A} \delta\left(\mu_{B} \mu_{T}^{\prime}+\beta k_{T}\right)-k_{A} \mu_{B} \mu_{T} \mu_{T}^{\prime}\right] \\
& -k_{A} \delta C \mu_{B}^{\prime} \mu_{T} \mu_{T}^{\prime}\left(\mu_{B} \mu_{T}^{\prime}+\beta k_{T}\right)-k_{A} \mu_{B} \mu_{B}^{\prime}\left(\mu_{T}\right)^{2}\left(\mu_{T}^{\prime}\right)^{2}-\alpha \beta \delta^{2} k_{B} k_{T} \mu_{T}^{\prime} C \\
c_{1}= & C \mu_{T} \mu_{T}^{\prime}\left\{\delta \mu_{A}\left[\mu_{B}\left(\gamma k_{T}-\mu_{B}^{\prime} \mu_{T}^{\prime}\right)-\mu_{B}^{\prime}\left(\mu_{B} \mu_{T}^{\prime}+\beta k_{T}\right)\right]\right. \\
& \left.+k_{A} \mu_{B} \mu_{B}^{\prime} \mu_{T} \mu_{T}^{\prime}-\alpha \beta \delta k_{B} k_{T}\right\} \\
c_{0}= & -\mu_{A} \mu_{B} \mu_{B}^{\prime}\left(\mu_{T}\right)^{2}\left(\mu_{T}^{\prime}\right)^{2} C .
\end{aligned}\right.
$$

A natureza e número de soluções biologicamente viáveis (todos os valores de $P$ positivos) são determinados por estes coeficientes.

Dada a impossibilidade de estudo analítico das soluções da equação (2.4), buscase soluções numéricas para o caso não-trivial. Porém, a partir do estudo realizado com a equação de antígenos [3], espera-se encontrar até dois pontos de equilíbrio nãotriviais, sendo um localmente e assintoticamente instável (L.A.I.), com densidade menor de antígenos $\bar{A}_{-}$, denominada break point, e outro, L.A.E., com concentração maior de antígenos $\bar{A}_{+}$, com $\bar{A}_{+}>\bar{A}_{-}$. No modelo onde a proliferação de células $B_{p}$ era mediada por antígenos, encontrou-se dois pontos de equilíbrio não-triviais com as características acima.

\section{Resultados Numéricos}

O estudo numérico é realizado com o conjunto de parâmetros apresentados na Tabela 1, os quais, em parte foram obtidos na literatura disponível, e para aqueles não especificados na literatura, estabeleceu-se uma correlação razoável com a homeostasia de células do corpo humano [1]. Os parâmetros do modelo têm as seguintes dimensões: para as taxas de mortalidades, $[d i a]^{-1}$, para produção de células do sistema imunológico, $[$ concentração $] \times[d i a]^{-1}$, para taxa de replicação de antígenos, [concentração $]^{-1} \times[\text { dia }]^{-1}$, e a capacidade de suporte $C$ é adimensional. Quanto, porém, às taxas de transição $\beta$ e $\delta$, à taxa de ligação antígeno-anticorpo $\alpha$ e à taxa de clonagem $\gamma$, elas são objeto de estudo mais cuidadoso neste trabalho, pois não existem dados referentes à interação entre sistema imunológico e parasitas. As dimensões destas taxas são $[\text { concentração }]^{-1} \times[\text { dia }]^{-1}$. Assim, o objetivo do estudo numérico é compreender a dinâmica dos pontos de equilíbrio, identificando-se aqueles parâmetros que mais a influenciam.

\begin{tabular}{|l|l|}
\hline$k_{A}=5[12]$ & $\mu_{A}=0,6$ \\
\hline$k_{B}=0,2$ & $\mu_{B}=0,05$ \\
\hline$k_{T}=0,9[12]$ & $\mu_{B}^{\prime}=0,2[12]$ \\
\hline$C=50$ & $\mu_{T}=0,03[12]$ \\
\hline & $\mu_{T}^{\prime}=1,2[10]$ \\
\hline
\end{tabular}

Tabela 1. Valores de parâmetros utilizados nas simulações. 
As simulações mostraram que $\beta$ e $\delta$ não são determinantes para a existência de soluções reais positivas para a equação (2.4), ou seja, uma vez fixados os demais parâmetros, observa-se que a referida equação possui duas raízes reais positivas independente dos valores atribuídos a $\beta$ e $\delta$. Todavia, $\alpha$ e $\gamma$ influenciam fortemente. Os estudos mostraram a existência de uma região de variação destes parâmetros, a saber, para $\beta=0,5$ e $\delta=0,5$ fixos, tem-se: para $\gamma=0,000001, \alpha \in(0 ; 69,4)$, e para $\alpha=0,001, \gamma \in(0 ; 0,26477)$. A correlação entre $\alpha$ e $\gamma$ é negativa quando a equação (2.4) possui duas raízes reais positivas e, ao mesmo tempo, resultam para as demais variáveis do ponto de equilíbrio, dadas pela equação (2.3), valores reais positivos, ou seja, quando $\alpha$ cresce, $\gamma$ decresce, e vice-versa. A correlação entre $\alpha$ e $\gamma$ é apresentada na Figura 1.

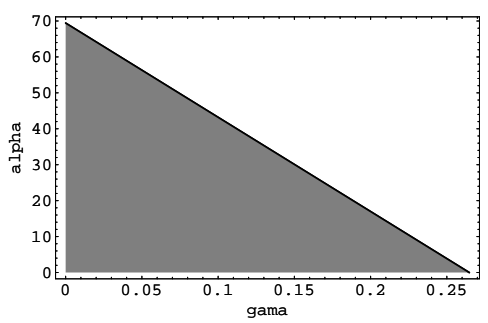

Figura 1: Correlação entre $\alpha$ e $\gamma$.

O comportamento das soluções da equação (2.4), diante da variação dos parâmetros $\alpha, \beta, \gamma$ e $\delta$, é mostrado na Figura 2, sendo que em cada caso tem-se um dos parâmetros variando e os demais fixos. Os parâmetros fixos utilizados foram: $\alpha=1,5, \beta=0,5, \gamma=0,15$ e $\delta=0,5$.

Como pode ser observado, se $\alpha$ e $\gamma$ estiverem na região apresentada na Figura 1, então há duas regiões possíveis para a inoculação inicial de antígenos. Se a concentração inicial estiver abaixo da curva inferior (Figura 2), a solução tenderá para a solução nula, ocasionando a eliminação do parasita, enquanto que, se estiver acima desta curva, a solução tenderá para a curva superior, que representa a instalação do parasita no hospedeiro.

Um fato relevante é que se $\gamma$ estiver fora do intervalo que permite a existência de duas raízes, o sistema possui apenas a solução trivial, independente da condição inicial. Observa-se com isso a existência de um valor limiar para a taxa de clonagem das células $B_{p}$, que será denominada $\gamma^{*}$, no exemplo, $\gamma^{*}=0,265$, tal que, se $\gamma>\gamma^{*}$, então o modelo apresenta apenas a solução de equilíbrio trivial, significando que o sistema imunológico é eficaz o bastante para eliminar todos os antígenos introduzidos, independente da inoculação inicial. E quando a taxa de clonagem está abaixo deste valor limiar, ou seja, $\gamma<\gamma^{*}$, existe a possibilidade da doença se estabelecer, uma vez que se tem a possibilidade da concomitância de antígenos (patógenos) com as células do sistema imunológico. E neste caso, se a concentração de antígenos ultrapassar $\bar{A}_{-}$, o sistema imunológico perde o controle e a doença se estabelece. 

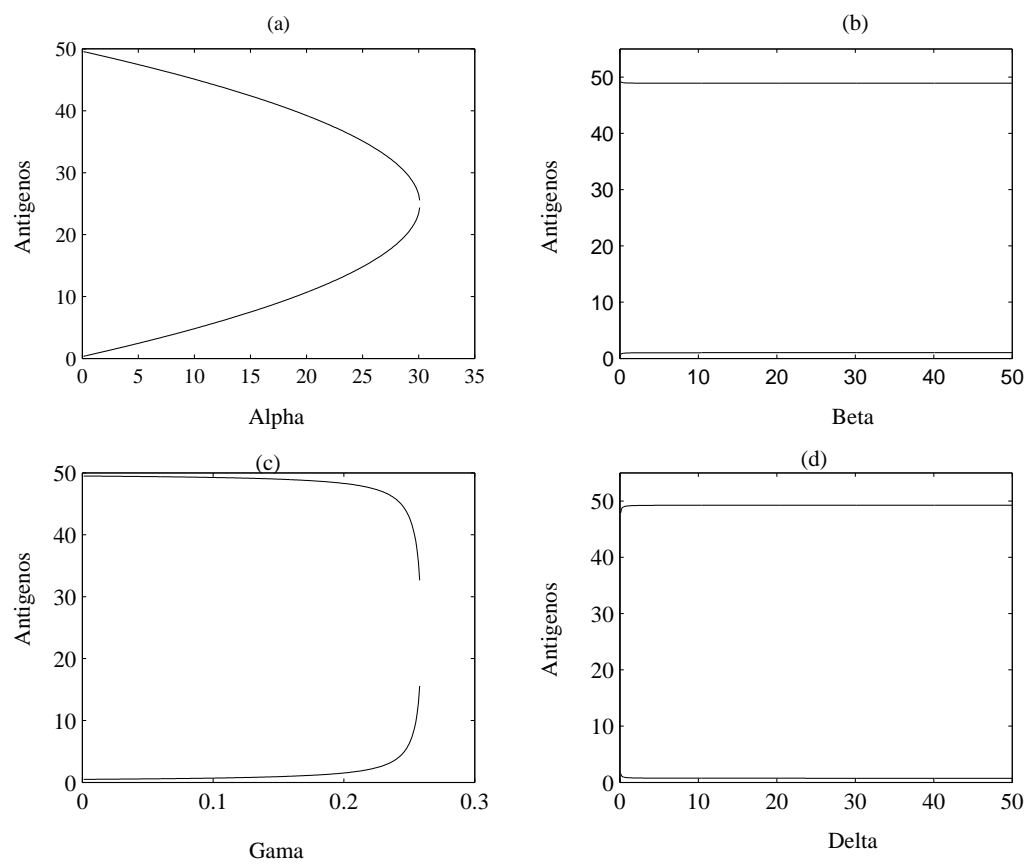

Figura 2: Comportamento das soluções com a variação dos parâmetros $\alpha, \beta, \gamma$ e $\delta$.

As duas soluções de equilíbrio não-triviais obtidas utilizando o conjunto de parâmetros apresentado na Tabela 1 , mais $\alpha=1,5, \beta=0,5, \gamma=0,15$ e $\delta=0,5$, são dadas por

$$
P_{-}=\left[\begin{array}{c}
\bar{A}_{-}=0,671 \\
\bar{B}_{-}=0,507 \\
\bar{B}_{p_{-}}=1,805 \\
\bar{T}_{-}=2,464 \\
\bar{T}_{a_{-}}=0,688
\end{array}\right] \quad \text { e } \quad P_{+}=\left[\begin{array}{l}
\bar{A}_{+}=49,265 \\
\bar{B}_{+}=0,471 \\
\bar{B}_{p_{+}}=2,013 \\
\bar{T}_{+}=0,037 \\
\bar{T}_{a_{+}}=0,749
\end{array}\right] .
$$

A estabilidade destas soluções foi estudada através da análise dos auto-valores associados à matriz Jacobiana nos dois pontos de equilíbrio $P_{-}$e $P_{+}$, cujos valores são

$$
P_{-}:\left[\begin{array}{l}
\lambda_{1}=3,225 \\
\lambda_{2}=-1,372 \\
\lambda_{3}=-0,123 \\
\lambda_{4}=-0,262-0,153 \times i \\
\lambda_{5}=-0,262+0,153 \times i
\end{array}\right] \quad \text { e } \quad P_{+}:\left[\begin{array}{l}
\lambda_{1}=-239,086 \\
\lambda_{2}=-24,662 \\
\lambda_{3}=-1,200 \\
\lambda_{4}=-0,425 \\
\lambda_{5}=-0,088
\end{array}\right]
$$

O ponto de equilíbrio $P_{-}$, que corresponde ao valor menor $\bar{A}_{-}$, tem $\lambda_{1}>0$, o que implica que $P_{-}$é instável [4]. Para o ponto de equilíbrio $P_{+}$, que corresponde ao 
valor maior $\bar{A}_{+}$, todos os autovalores são reais distintos e negativos, sendo, portanto, $P_{+}$, um ponto de equilíbrio hiperbólico estável (nódulo).

Estes resultados mostram a existência de um atrator não-trivial para o modelo $\left(P_{+}\right)$, no qual o valor da variável $A$, no equilíbrio dado por $\bar{A}_{+}$, é muito próximo da capacidade de suporte do meio $(C)$ (para os parâmetros utilizados), e a existência de um ponto de equilíbrio instável $\left(P_{-}\right)$, que é tido como break point [8]. O valor da variável $A$ neste break point, no equilíbrio dado por $\bar{A}_{-}$, representa um valor limiar para a eliminação do parasita ou sua instalação no organismo do hospedeiro, uma vez que, pelo fato de $P_{-}$ser instável, uma pequena perturbação que diminua a concentração de antígenos faz com que a solução convirja para a trivial e uma pequena perturbação que aumente a sua concentração faz com que a solução convirja para $P_{+}$, ou seja, depende da condição inicial. Isso pode ser visualizado na Figura 3, que representa o comportamento dinâmico da variável $A$ considerando uma pequena perturbação em torno de seu valor, no ponto $P_{-}$. Figura 3 foi obtida novamente tomando os parâmetros apresentados na Tabela 1 , mais $\alpha=1,5, \beta=0,5, \gamma=0,15$ e $\delta=0,5$, e condições iniciais dadas por $A(0)=0,67$ em (a) e $A(0)=0,671 \mathrm{em}$ (b).

(a)

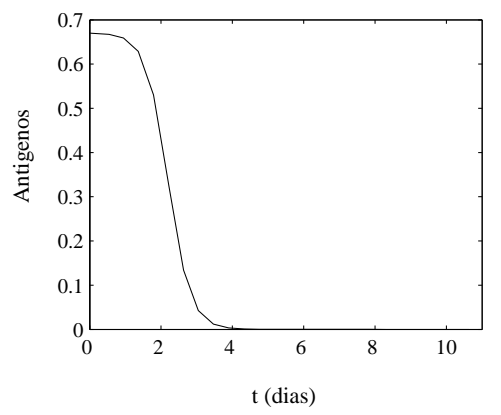

(b)

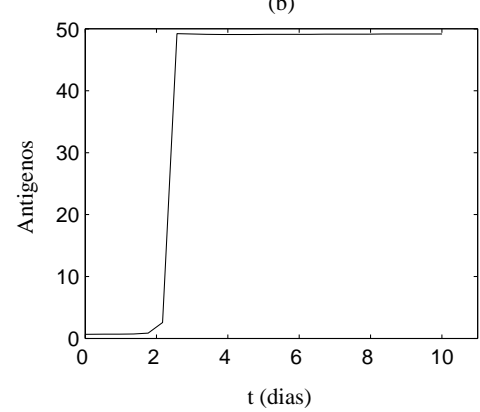

Figura 3: Dinâmica dependente de condição inicial.

\section{Conclusão}

Neste trabalho estudou-se um modelo matemático que descreve a ação do sistema imunológico mediante a detecção de um patógeno circulante na corrente sanguínea ou tecidos extra-celulares. O estudo mostrou que a existência das soluções nãotriviais, as quais expressam a presença do micro-organismo no hospedeiro, depende da ação de anticorpos via interação das células $B-T$, seguida de sua interferência na eliminação do antígeno. O modelo proposto mostrou ter três soluções de equilíbrio estacionários, sendo uma trivial (ausência de antígeno e sistema imune em repouso), a qual é L.A.E. independentemente dos parâmetros, e duas não-triviais, dependente 
fortemente dos parâmetros que representam, respectivamente, a taxa de clonagem das células $B$ plasma e a taxa de eliminação de antígenos devido à ação do sistema imunológico. As taxas de ativação das células $B$ e $T$ não se mostraram muito relevantes para a existência de soluções não-triviais. Do ponto de vista biológico, estes resultados indicam que se a taxa de clonagem das células plasma (que produzem anticorpos) estiverem acima de um determinado patamar, o sistema imunológico é capaz de debelar qualquer quantidade de antígeno que seja introduzido no organismo. Caso contrário, o controle depende da introdução inicial, ou seja, se esta for tal que a concentração de antígenos (após o sistema ser ativado) fique abaixo do valor determinado pela solução instável, então a eliminação do antígeno estará garantida. Por outro lado, se a concentração inicial de antígenos for tal que, após a ativação do sistema imunológico, ela esteja acima da solução instável, então o sistema falhará e o patógeno se multiplicará até atingir uma concentração próxima à capacidade suporte do meio.

\begin{abstract}
In this paper we present a mathematical model in order to explain the immune system's response taking into account the production of antibodies by B cells, which are activated by $\mathrm{T}$ cells, when a parasite (antigen) invades the human body. The analysis of the steady states of the model shows the existence of a region regarded to the set of the values of parameters in which three equilibrium points are biologically viable. Two of them are stable, which represent, respectively, the parasite elimination or absence (trivial solution) and the persistence of the parasite which major concentration, and the third is unstable, a non-trivial equilibrium which minor concentration of antigens, called "break point". Out of this region only the trivial solution is biologically viable. This shows that the immune system's response depends both on its state (which depends on genetic, nutritional, nervous system, etc.) and on the inoculated antigen concentration, which agrees with that observed in practice.
\end{abstract}

\title{
Referências
}

[1] A.K. Abbas, A.H. Lichtman e J.S. Pober, "Imunologia Celular e Molecular", Revinter Ltda., $3^{a}$ Ed., Rio de Janeiro, 2000.

[2] S. Barrozo, H.M. Yang e C.H. Dezotti, Uma abordagem matemática em imunologia, em "Matemática Aplicada à Fisiologia"(H.M. Yang, R. Sampaio e A. Sri Ranga, eds.), SBMAC, São Carlos, pp. 93-117, 2003.

[3] S. Barrozo e H.M. Yang, Desenvolvimento de um modelo para resposta imunológica primária célula-mediada, Tendências em Matemática Aplicada e Computacional, 7, No. 1 (2006), 31-41.

[4] L. Edelstein-Keshet, "Mathematical Models in Biology", Random House, New York, 1987.

[5] C.A. Janeway Jr., How the immune system recognizes invaders. Scientific American, 269 (1993), 73-79. 
[6] C.A. Janeway, P. Travers, M. Walport, e J.D. Capra, "O Sistema Imunológico na Saúde e na Doença", Artmed Ed., Porto Alegre, 2000.

[7] P. Marrack e J.W. Kappler, How the immune system recognizes the body, Scientific American, 269 (1993), 81-89.

[8] R.M. May, Togetherness among schistosome: its effecgts on the dynamics of the infection, Math. Biosc., 35 (1977), 301-343.

[9] G.J.V. Nossal, Life, death and the tmmune system, Scientific American, 269 (1993), 53-62.

[10] M. Oprea e A.S. Perelson, Exploring the machanisms of primary antibody responses to T-cell dependent antigens, J. Theor. Biol., 181 (1996), 215-236.

[11] W.E. Paul, Infectious diseases and the immune system, Scientific American, 269 (1993), 91-97.

[12] A.S. Perelson e P.W. Nelson, Mathematical analysis of HIV-I dynamics in vivo, Society for Industrial and Applied Mathematics, 41 (1999), 3-44.

[13] I.L. Weissman e M.D. Cooper, How the immune system develops, Scientific American, 269 (1993), 65-71. 